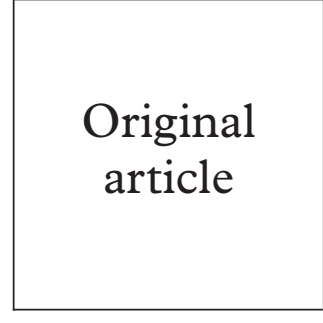

\title{
Impact of menstrual cycle on the diagnostic performance of LCR, TMA, and PCE for detection of Chlamydia trachomatis in home obtained and mailed vaginal flush and urine samples
}

\author{
J K Møller, B Andersen, F Olesen, T Lignell, L Østergaard
}

Objectives: To assess the impact of the menstrual cycle on the diagnostic performance of various assays for detection of Chlamydia trachomatis in home obtained and mailed vaginal flush and urine specimens.

Methods: A ligase chain reaction assay (LCR; Abbott Laboratories), a transcription mediated amplification assay (TMA; Gen-Probe), and an enzyme amplified immunoassay (PCE; Dako Diagnostics) were evaluated for their validity in detecting $C$ trachomatis in vaginal flush, first void urine, and midstream urine specimens obtained by female high school students at home and mailed directly to the diagnostic laboratory.

Results: $C$ trachomatis was detected in 45 of 889 females (5.1\%). The vaginal flush material was positive by TMA and LCR in $84 \%$ and $82 \%$ of the chlamydia positive females, respectively. First void urine was positive by TMA in $73 \%$ and by LCR in $49 \%$ of the cases. Midstream urine was positive by TMA and LCR in $69 \%$ and $42 \%$ of the females, respectively. On a pool of first void and midstream urine, PCE detected $49 \%$ of the chlamydia positive females. The overall prevalence of $C$ trachomatis increased with increasing time after the last menstrual bleeding. In urine samples, but not vaginal flush specimens, obtained 3 weeks after the last menstrual bleeding, the sensitivities of TMA, LCR, and PCE decreased markedly suggesting that inhibitors to the assays are excreted in the urine but not in vaginal secretions at this time.

Conclusion: Vaginal flush samples are superior to urines for detection of chlamydia infections in females. In screening of young asymptomatic females, samples should be obtained in the latter part of the menstrual cycle.

(Sex Transm Inf 1999;75:228-230)

Keywords: vaginal flush specimens; urine; menstrual cycle; Chlamydia trachomatis

\section{Introduction}

More samples from asymptomatic women are submitted for detection of Chlamydia trachomatis by offering a screening strategy implying home obtained and mailed samples. ${ }^{1-3}$ This makes it very important to assess which sample type and assay should be used for optimal diagnostic performance. Owing to the lower burden of $C$ trachomatis organisms in asymptomatic females, the diagnostic tests may be more susceptible to hormonally mediated inhibitors. ${ }^{4}$ It is therefore also important to assess the optimal time of collection with regard to menstrual cycle, since this can be predetermined in screening programmes targeting asymptomatic individuals.

In this study, the diagnostic performance of two nucleotide amplification methods (TMA and LCR) and one enzyme amplified immunoassay (PCE) on self obtained and mailed urine and vaginal flush samples was evaluated and correlated with the time elapsed since last menstrual bleeding.

\section{Methods}

STUDY POPULATION

An intervention study group of 2603 female high school students participating in a larger study $^{3}$ addressing 8909 female and male students from 17 high schools in Aarhus County were collectively informed by one of the investigators (using the same set of slides) at the school about chlamydia infections and provided with a kit for collecting samples at home. Samples were mailed to the laboratory from 889 female students. The mean age of the students was 17.9 years (SD 1.5 years). The kit comprised a pipette containing $5 \mathrm{ml}$ of sterile isotonic sodium chloride for vaginal flush, and two $30 \mathrm{ml}$ sterile sample tubes for obtaining a first void urine and a midstream urine. Included were also written instructions as previously described, ${ }^{1}$ a request form, a questionnaire, and a stamped envelope for mailing the samples to the department of clinical microbiology. The study was approved by the local ethics committee and both students and their parents were informed about the study by letter. Mailing samples to the laboratory implied informed consent.

DIAGNOSTIC TESTS

The following tests were used; (1) the ligase chain reaction assay (LCR; Abbott Laboratories, Chicago, IL, USA), (2) the transcription mediated amplification assay (TMA; Gen- 
Table 1 Detection of $C$ trachomatis in 862 females by week after the menstrual bleeding

\begin{tabular}{|c|c|c|c|c|c|c|c|c|}
\hline \multirow[b]{3}{*}{ Week } & & & & \multicolumn{5}{|c|}{ Number of females positive in } \\
\hline & \multicolumn{3}{|c|}{ Number of females } & \multicolumn{3}{|l|}{ Urine } & \multicolumn{2}{|c|}{ Vaginal flush } \\
\hline & Total & Pos & $(\%)$ & LCRt & $T M A \dagger$ & $P C E \ddagger$ & $L C R$ & $T M A$ \\
\hline 1 & 304 & 11 & (3.6) & $7(64) \S$ & $9(82)$ & $8(73)$ & $10(91)$ & $10(91)$ \\
\hline 2 & 209 & 11 & (5.3) & $6(55)$ & $10(91)$ & $4(36)$ & $9(82)$ & $10(91)$ \\
\hline 3 & 214 & 11 & $(5.1)$ & $4(36)$ & $6(55)$ & $3(28)$ & $9(82)$ & $9(82)$ \\
\hline $4+$ & 135 & 10 & (7.4) & $5(50)$ & $8(80)$ & $6(60)$ & $7(70)$ & $8(80)$ \\
\hline
\end{tabular}

${ }^{\star}$ Based on the expanded reference standard; ffirst void; łmixed first void and midstream Spercentage of the positive females in parentheses.

Probe Inc, San Diego, CA, USA), (3) an enzyme amplified immunoassay (PCE; Dako Diagnostics, Denmark), and (4) a PCR (Roche Amplicor Chlamydia trachomatis, Basle, Switzerland).

SPECIMEN PROCESSING

Aliquots of all specimens were made and stored at $-80^{\circ} \mathrm{C}$.

To reduce costs and since pooling of samples does not affect sensitivity and specificity of molecular amplification assays for detection of $C$ trachomatis, ${ }^{5}$ samples from individuals (vaginal flush, first void urine, and midstream urine) were pooled for initial testing.

For LCR, a pool consisting of $1 \mathrm{ml}$ of first void urine, $1 \mathrm{ml}$ of midstream urine, and $1 \mathrm{ml}$ of vaginal flush material was made. For TMA, a pool consisting of $1.5 \mathrm{ml}$ of first void urine, $1.5 \mathrm{ml}$ of midstream urine, and $1 \mathrm{ml}$ of vaginal flush material was made. The amount of material of the individual specimens in the pool was chosen according to the recommendations of the manufacturer for the assay on urine. For PCE, a pool consisting of $5 \mathrm{ml}$ of first void urine and $5 \mathrm{ml}$ of midstream urine only was made.

For patients having a positive pool, the frozen aliquots of the individual specimen types (urines and vaginal flush material) were subsequently examined by the LCR and the TMA assay according to the assay package insert for urine specimens and as described earlier. ${ }^{16}$ Confirmatory testing of divergent results was made with PCR on $5-7 \mathrm{ml}$ of urine. $^{7}$

DEFINITION OF INFECTION

Females were designated infected if any two of the four assays (LCR, TMA, PCE, and PCR) yielded a positive result on at least one of the three specimen types or pools.

MENSTRUAL CYCLE

From a precoded choice of week 1 to week $4+$ on the request form, participants were asked to mark the period of time elapsed since the last day of the menstrual bleeding.

STATISTICAL ANALYSIS

The $\chi^{2}$ test for trend was used to ascertain the $\mathrm{N} \times 2$ table and Fisher's exact test for the fourfold table.

\section{Results}

Forty five females $(45 / 889 ; 5.1 \%)$ were positive for $C$ trachomatis as defined by the expanded reference standard. The sensitivity of the sepa- rate assays on the individual sample material from the 45 chlamydia positive participants were (95\% CI in parentheses): LCR and TMA on first void urine: $49 \%(34-65 \%)$ and $73 \%$ (58-85\%), respectively; LCR and TMA on midstream urine $42 \%(28-58 \%)$ and $69 \%$ (53-82\%), respectively; LCR and TMA on vaginal flush: $82 \%(68-92 \%)$ and $84 \%$ (71$94 \%$ ), respectively; PCE on mixed urine: $49 \%$ (34-65\%).

The females were asked about the acceptability of the home sampling procedures. To the question "was it unpleasant to obtain the urine samples," the answers were "no" $(75 \%)$, "slightly" (17\%), "rather" (3\%), "very" (1\%), and "not indicated" (4\%). For vaginal flush samples the corresponding answers were "no" (43\%), "slightly" (38\%), "rather" (11\%), "very" (4\%), and "not indicated" (4\%).

Data on menstrual cycle were obtained from 862 of the 889 participants including 43 of 45 chlamydia positive females. The proportion of chlamydia positive females was not equal for the various weeks of the menstrual cycle (table 1 ). The percentage of positive females thus increased from $3.6 \%(11 / 304)$ in the first week to $9.0 \%(8 / 89)$ in the fourth week after menstrual bleeding (one tailed $\mathrm{p}=0.04$ ) or $7.4 \%$ for week $4+$ (one tailed $\mathrm{p}=0.06$ ). In contrast with the increasing trend in overall chlamydia positives from week to week, the examination of urines (table 1) showed that a significant lower number of infected females was detected by LCR, TMA, and the PCE assay in the third week after menstrual bleeding compared with the other weeks in the menstrual cycle (one tailed $\mathrm{p}=0.008$ ). This latter difference in number of positives between weeks of the menstrual cycle was not seen for vaginal flush samples (table 1).

\section{Discussion}

Universal screening of large low prevalence groups for infection with $C$ trachomatis is dependent on sensitive assays and non-invasive procedures for specimen sampling. ${ }^{138}$ Urine has been the most widely used self collectable sample material, ${ }^{16}$ but several detection methods have a poor performance on urine. ${ }^{9}$ The present study includes three types of sampling material and three different methods for detection of $C$ trachomatis. An expanded reference standard was used to make the evaluation of the individual assays less biased than by comparing two assays and performing discrepancy analyses. ${ }^{10}$

The LCR and the TMA assay consistently found the same proportion of chlamydia positive vaginal flush samples, whereas the LCR detected markedly fewer positive urines than TMA. Inhibitors in the urine to LCR may explain the low sensitivity. ${ }^{11}$ Dilution of the urine or a wash of the urine before performing the LCR test may improve the results $(67 \%$ of the initial false negative samples became positive by this procedure in our study; data not shown). The enzyme amplified immunoassay (PCE) was performed on mixed urines only and detected about half of the chlamydia infected females. 
The fact that the percentage of positive females was markedly higher during the fourth week after the menstrual bleeding supports a previous observation of a hormonal influence on growth of $C$ trachomatis. ${ }^{412}$ However, a significant inhibition of both LCR, TMA, and PCE was seen on urines in the third post menstrual week. This phenomenon was also observed by Horner et al. ${ }^{4}$ Inhibition was not seen for vaginal flush samples which suggests that the menstrual cycle factors are excreted in the urine and not present in vaginal secretions. This corroborates a similar difference between urine and non-urine specimens seen by Horner et $a l^{4}$ examining the influence of the menstrual cycle on the detection of $C$ trachomatis in cervical swabs and urines. We have unpublished data indicating an inhibition of the chlamydia detection by LCR during midcycle in chlamydia spiked urines obtained during the menstrual cycle from a group of females taking oral contraceptives compared with a group of females not using oral contraceptives. This may suggest an inhibition by oestrogen in the urine as the oral contraception raises the level of oestrogen in midcycle. However, further studies are needed because of the small number of females examined (18 people).

Specimens were mailed uncooled to the laboratory and with a few exceptions received in the laboratory the day after. These mailing conditions seem not to make vaginal flush or urine inferior to conventional swabs taken by a general practitioner. ${ }^{1}$ It may therefore be advantageous to replace the routine cervical swab for chlamydia with a self obtained vaginal flush sample collected at home. We conclude that vaginal flush samples in general are better than urines for detection of chlamydia infections in females, and that in screening of young asymptomatic females, samples should be obtained in the latter part of the menstrual cycle.

We are grateful to Gitte Høj, Jannie Thor, and Mette Jensen for excellent technical assistance. We thank Dako Diagnostics and
Gen-Probe (Kingo Diagnostika, Denmark) for providing the PCE and the TMA kits, respectively.

Contributors: JKM initiated and coordinated the formulation of the laboratory study, discussed core ideas, participated in the design of the clinical protocol and in informing the students, performed data collection, analyses, interpretation, and writing of the paper; he will act as guarantor for the paper. BA discussed core ideas, participated in protocol design, information process, data collection and interpretation, and edited the paper. FO discussed core ideas, participated in protocol design, information process, interpretation of data, and edited the paper. TL coordinated the experiments with chlamydia spiked urines during menstrual cycle, participated in protocol design, and edited the paper. LØ initiated and coordinated the formulation of the clinical study hypothesis and the core ideas, obtained approval from the ethics commitee and the Danish Data Protection Agency, designed the clinical protocol, participated in the inforAgency, designed the clinical protocol, participated in the infor-
mation process, data collection and interpretation, and edited the paper.

Conflict of interest: None.

1 Østergaard L, Møller JK, Andersen B, et al. Diagnosis of urogenital Chlamydia trachomatis infection in women based on mailed samples obtained at home: multipractice comparative study. BMF 1996;313:1186-9.

2 Andersen B, Østergaard L, Møller JK, et al. Home sampling versus conventional contact tracing for detecting Chlamydia trachomatis infections in male partners of infected women: randomised study. BMF 1998;316:350-1

3 Østergaard L, Andersen B, Olesen F, et al. Efficacy of home sampling for screening of Chlamydia trachomatis infections: randomized study. BMF 1998;317:26-7.

4 Horner PJ, Crowley T, Leece J, et al. Chlamydia trachomatis detection and the menstrual cycle. Lancet 1998;351:341-2.

5 Peeling RW, Toye B, Jessamine P, et al. Pooling of urine specimens for PCR testing: a cost saving strategy for Chlamydia trachomatis control programmes. Sex Transm Inf 1998;74:66-70.

6 Mouton JW, Verkooyen R,Van der Meijden WI, et al. Detection of Chlamydia trachomatis in male and female urine specimens by using the amplified Chlamydia trachomatis test. I Clin Microbiol 1997:35:1369-72.

7 Møller JK, Østergaard L, Hansen JT. Clinical evaluation of four non-related techniques for detection of Chlamydia trachomatis in endocervical specimens obtained from a low prevalence population. Immun Infect Dis 1994;4:191-6.

8 Hook III EW, Smith K, Mullen C, et al. Diagnosis of genitourinary Chlamydia trachomatis infections by using the ligase chain reaction on patient-obtained vaginal swabs. $f$ Clin Microbiol 1997;35:2133-5.

9 Chernesky MA, Jang D, Lee H, et al. Diagnosis of Chlamydia trachomatis infections in men and women by testing first-void urine by ligase chain reaction. $f$ Clin Microbiol 1994;32:2682-5.

10 Hadgu A. The discrepancy in discrepant analysis. Lancet 1996;348:592-3.

11 Berg ES, Ånestad G, Moi H, et al. False-negative results of a ligase chain reaction assay to detect Chlamydia trachomatis due to inhibitors in urine. Eur f Clin Microbiol Infect Dis 1997; 16:727-31.

12 Jensen IP, Thorsen P, Møller BR. Sensitivity of ligase chain reaction assay of urine from pregnant women for Chlamydia trachomatis. Lancet 1997;349:329-30. 